CAHIERS DE

NARRATOLOGIE

\section{Cahiers de Narratologie}

Analyse et théorie narratives

32 | 2017

Récit et argumentation, interactions, lieux et dispositifs sociaux

\title{
Récits des élèves et récits des scientifiques dans les sciences de la nature
}

Denise Orange Ravachol

\section{(2) OpenEdition \\ Journals}

Electronic version

URL: http://journals.openedition.org/narratologie/7838

DOI: 10.4000/narratologie.7838

ISSN: 1765-307X

Publisher

LIRCES

\section{Electronic reference}

Denise Orange Ravachol, «Récits des élèves et récits des scientifiques dans les sciences de la nature », Cahiers de Narratologie [Online], 32 | 2017, Online since 21 December 2017, connection on 01 May 2019. URL : http://journals.openedition.org/narratologie/7838; DOI : 10.4000/narratologie.7838

This text was automatically generated on 1 May 2019.

Article L.111-1 du Code de la propriété intellectuelle. 


\title{
Récits des élèves et récits des scientifiques dans les sciences de la nature
}

\author{
Denise Orange Ravachol
}

\section{Introduction : sciences et récits}

1 Les relations entre sciences et récits sont complexes et discutées quand on sait la tendance commune de mettre en histoire les expériences et les problèmes du monde. Selon Bruner (1996, pp. 151-152), la forme narrative est la manière la plus naturelle et la plus précoce que nous avons d'organiser les premières, de détecter et de prendre en charge les seconds. "L'histoire, écrit-il, est extrêmement sensible à tout ce qui contrarie notre sens du "normal" (2002, p. 17). Or la prétention de l'activité scientifique est également de prendre en charge des problèmes d'explication du monde (Popper, 1985, pp. 192-193; Jacob, 1981, p. 29). Et de le faire de manière rationnelle. Quelles parentés ou dissemblances peut-on établir entre pensées commune et scientifique, dont les jeux respectifs semblent concurrentiels? C'est à cette question que nous consacrons cet article, en nous focalisant sur quelques problèmes des sciences de la vie et de la Terre, sans opposer a priori récit populaire et explication scientifique, sans non plus les confondre. Au travers de ce que font les scientifiques et de ce que produisent spontanément des élèves, autrement dit « les mises en histoires » qu'ils effectuent, nous tentons de faire ressortir leurs caractéristiques.

\section{Récit, problématisation et construction de savoirs scientifiques}

2 Nous plaçons notre étude dans un cadre rationaliste (Bachelard, Popper, Canguilhem). La construction de savoirs scientifiques, qu'il s'agisse des scientifiques ou des élèves, impose de porter une attention particulière aux problèmes et à leur travail, dans une logique de 
clarification de ce qu'il s'y joue au regard du sens commun. Cette approche est exigeante car l'activité scientifique vise avant tout le développement du « sens du problème » (Bachelard, 1938, p .14), notamment par mise en jeu de la critique (Popper, 1985, p.191); et avec l'assise forte que la connaissance scientifique est toujours à considérer comme «la réforme d'une illusion » (Bachelard, 1971, p. 12).

\section{Le sens du problème}

3 Les récits participent fondamentalement à la pensée humaine : "C'est le médium que nous préférons, pour le meilleur et pour le pire » écrit Bruner (2002, p.79). Ils domestiquent en effet l'inattendu et l'exceptionnel, ils assemblent le disparate et le fragmentaire (Adam, 1984), ils donnent forme et sens au monde qui nous entoure (Bruner, 1997) : «la fonction de l'histoire est de trouver une intention qui atténue ou au moins rende compréhensible une déviation par rapport à un élément culturel canonique» (Ibidem, p. 63). Les récits semblent donc relever d'une prédisposition humaine et témoigner de notre capacité à détecter les problèmes avant même de représenter une tentative de leur résolution: "Un grand récit nous invite à poser des problèmes; il n'est pas là pour nous dire comment les résoudre. Il nous parle d'une situation de crise, du chemin à parcourir et non du refuge auquel il mène » (Bruner, 2002, p. 22). Voici qui vaudrait déjà un rapprochement entre pensées commune et scientifique.

\section{La réforme d'une illusion}

4 Comme les ressources narratives d'une culture (contes, vieilles histoires, littérature, potins...; Bruner, 2002, p. 82), l'activité scientifique comporte sa part de fictions. Popper (1985, p. 191) établit une filiation entre mythe et science. Mais il ajoute que ce que nous appelons « science » se distingue des mythes non par la forme (il s'agit bien toujours de raconter une histoire qui explique un phénomène) mais parce qu'il s'y ajoute un corrélat, la prise en compte critique de cette histoire par la discussion et l'empirie. Ce faisant, il dégage le savoir scientifique de la connaissance commune, fortement subjective, pour l'installer dans un troisième monde, "un monde sans sujet connaissant ", un monde objectif et autonome, dont les « habitants » sont les systèmes théoriques, les problèmes et états de ces problèmes (état des discussions, état des échanges d'arguments critiques) (Popper, 1991).

5 Longtemps avant l'apparition de la critique, il y avait déjà développement de la connaissance - de la connaissance incorporée dans le code génétique. Le langage permet la création et la mutation des mythes explicatifs, ce que le langage écrit est venu plus tard renforcer. Mais seule la science remplace l'élimination de l'erreur dans la lutte violente pour la vie par la critique rationnelle non violente; seule elle nous permet de remplacer le meurtre (monde 1) et l'intimidation (monde 2) par les arguments impersonnels du monde 3. (Popper, 1991, p. 151)

6 Ainsi défini, le troisième monde relève d'une saisissante dynamique tensionnelle : il est un produit de l'activité humaine qui paradoxalement libère les hommes et se libère des hommes, tout en agissant en retour sur eux. L'activité scientifique, en cherchant à expliquer le premier monde (le monde "réel») apparaît alors comme le passage sans cesse renouvelé du deuxième monde, c'est-à-dire du monde du sujet qui la pratique (le chercheur en géologie, l'élève) au monde du savoir scientifique, problématique et raisonné, dont l'épaisseur tient à ce qu'il donne à voir du travail des problèmes et de la 
construction argumentée des solutions. Ce troisième monde comporte à la fois des éléments théoriques (modèles, principes, modes de raisonnement) mais aussi des faits construits (contraintes empiriques), les deux existant en interaction. Il ne se limite pas aux solutions des problèmes.

7 Si donc nous concevons la science comme une entreprise humaine et culturelle, le récit en est forcément partie prenante. Mais il s'agit d'en faire « un usage responsable » (Bruner, 1996, p. 119), de s'assurer qu'il garde la vivacité du problème, de lui donner une épaisseur critique.

\section{Des modes de raisonnement plus élaborés}

Parce qu'étroitement contrôlés par la critique, les récits explicatifs qu'invente la pensée scientifique s'affranchissent d'un empirisme immédiat (ce qui n'empêche pas cependant d'avoir des conceptions empiristes). En se racontant des histoires, la pensée commune se démarque également d'un empirisme immédiat, sans toutefois se défaire des croyances personnelles caractéristiques du deuxième monde de Popper. Bruner souligne bien que "lorsque nous inventons les mondes possibles de la fiction, nous ne quittons jamais vraiment l'univers qui nous est familier» (Bruner, 2002, p. 82). Les récits scientifiques s'étoffent aussi par la complexité des modes de raisonnement et des formes de temps qu'ils mobilisent. La construction de savoirs scientifiques relève ainsi d'une véritable extraction de la pensée commune, terriblement efficace par les économies de raisonnement qu'elle réalise (causes ad hoc, causalité simple par exemple): «dans la connaissance vulgaire, les faits sont trop tôt impliqués dans des raisons. Du fait à l'idée, le circuit est trop court » (Bachelard, 1938, p. 44). Il reste à repérer tout à la fois le complexe et le parcimonieux de la pensée scientifique.

\section{Les textes du savoir scientifique}

9 Le mode narratif de l'explication tient donc un rôle essentiel dans l'élaboration de la rationalité scientifique: "l'activité scientifique est faite de la construction et de la défense de points de vue d'abord fictionnels qui sont parfois transformés en faits stabilisés » (Latour \& Woolgar, 1988, p. 249). De plus, il se concrétise par une mise en texte des savoirs. Le lent processus de maturation de ces textes mobilise des inscriptions variées qui se superposent. Il extrait du champ de la controverse des énoncés stabilisés, manœuvre sur ces énoncés pour empêcher leur destruction par des énoncés alternatifs ou, si c'est le cas, pour être à l'origine d'un isolement de leur auteur. Les textes de savoirs scientifiques ont alors une épaisseur où s'entrecroisent des récits (la situation de la recherche par rapport à d'autres travaux, les comptes rendus d'expérimentation, etc.) et des intrigues différents. Sous peine d'être infinis, leur élaboration comporte nécessairement des réifications, la construction de boîtes noires, la neutralisation de petites intrigues. Il faut donc voir le texte du savoir comme l'émergence d'un nouveau récit, plus universel et avec des lacunes contrôlées.

\section{Conclusion}

10 La construction de savoirs scientifiques mobilise une heuristique narrative marquée par la transformation de récits du sens commun (de «petites histoires») en récits 
explicitement associés au travail critique de problèmes et constitutifs de savoirs raisonnés (apodictiques). Nous parlons de problématisation pour désigner le processus qui y conduit (Fabre \& Orange, 1997 ; Orange Ravachol, 2012). Dans la comparaison de ce que produisent les scientifiques et les élèves, nous devons repérer ce qui marque le passage de récits de type "petites histoires" en savoirs scientifiques. Deux types de repères vont nous servir.

11 a. Le passage de l'intra-objectal à l'inter-objectal

En référence à Piaget \& Garcia (1983, p.303), nous considérons que la construction de connaissances gagne en scientificité dès lors que l'on passe d'une centration sur les objets et leurs propriétés pour une focalisation sur les relations entre les objets. Une approche intra-objectale, typique des "petites histoires », repose sur " la découverte d'un ensemble de propriétés dans les objets ou les évènements, mais sans autres explications que locales et particulières » (ibidem, p. 303). L'approche inter-objectale, quant à elle, valorise les mises en relation des objets entre eux et la recherche des transformations reliant les objets selon de multiples formes de correspondances. Elle va avec la définition de systèmes plus complexes et la mobilisation de modes de raisonnement plus élaborés.

\section{b. Une diversification des modes de raisonnement}

Alors que l'intra-objectal favorise des explications basées sur les propriétés d'objet, la causalité et la succession temporelle simples («donc/et puis») portées jusqu'au syncrétisme, l'inter-objectal ouvre dans certains domaines sur la considération de flux entre compartiments (des entrées/sorties) et la simultanéité de leur réalisation («en même temps ») autrement dit sur des raisonnements systémiques. Ce recours à des manières de pensée « extra-ordinaires » participent grandement de la caractérisation du fonctionnement des systèmes tels que la science les aborde (Astolfi, 2008, p. 17).

13 Ainsi nous disposons d'outils pour interroger, dans quelques problèmes des sciences de la nature (biologie et géologie) et en comparaison de ce que font les scientifiques, les « mises en histoire » ordinaires des élèves et les formes de dépassement de récits qu'ils doivent effectuer pour construire des savoirs scientifiques. De par les types de problèmes caractéristiques des sciences de la nature, des problèmes fonctionnalistes et des problèmes historiques, deux sortes de dépassement des récits ordinaires sont envisagées. Nous les caractérisons en nous basant sur les productions langagières écrites et orales des scientifiques et des élèves, et recherchons ce qu'elles sous-tendent d'approche des systèmes, de formes de raisonnement, et d'argumentations.

\section{Première sorte de dépassement de récits ordinaires en sciences : l'appropriation de modes de pensée nouveaux}

\section{Systèmes biologiques et géologiques et stabilité structurelle}

La première sorte de dépassement des récits ordinaires que nous identifions se rattache à des problèmes de fonctionnement (problème fonctionnaliste) de systèmes biologiques et géologiques (le système cardio-vasculaire humain ; la dorsale océanique) en régime « de croisière ». Ces systèmes sont alors dans un état de stabilité dynamique : ils changent tout en ne changeant pas. En effet, ils conservent dans le temps leurs formes et leurs caractéristiques, malgré les flux de matières qui les traversent et les perturbations qui les 
affectent. Ainsi les paramètres caractéristiques du système sanguin (glycémie, etc.) se maintiennent-ils dans une certaine fourchette de valeur, alors que des entrées et des sorties de matières se produisent en permanence; et pour ce qui est des dorsales océaniques, leur constitution et leur organisation restent inchangées parce que ce qu'elles perdent en matériaux du fait de la dérive des deux plaques qu'elles délimitent est compensé par des ajouts de matériaux équivalents issus de la solidification de magmas mantelliques. Le travail de ces problèmes exige donc des élèves qu'ils adoptent un raisonnement systémique, et qu'ils rompent avec les « petites histoires » explicatives (de nutriments et de déchets pour le système cardio-vasculaire; de plaques pour la dorsale) qu'ils proposent de manière fréquente et récurrente.

\section{Approvisionnement des organes en substances nutritives et élimination de leurs déchets}

Si nous proposons à des lycéens de s'intéresser au fonctionnement des organes, les muscles en particuliers, c'est aisément qu'ils se remémorent leurs besoins (oxygène issu de l'air, nutriments comme le glucose en provenance de l'alimentation) et les "déchets » qu'ils produisent (dioxyde de carbone éliminé au niveau des poumons, déchets azotés éliminés dans l'urine). Ils construisent sans peine le schéma de la double circulation du sang (figure 1) expliquant l'approvisionnement en dioxygène du muscle et ses rejets et évacuation de dioxyde de carbone.

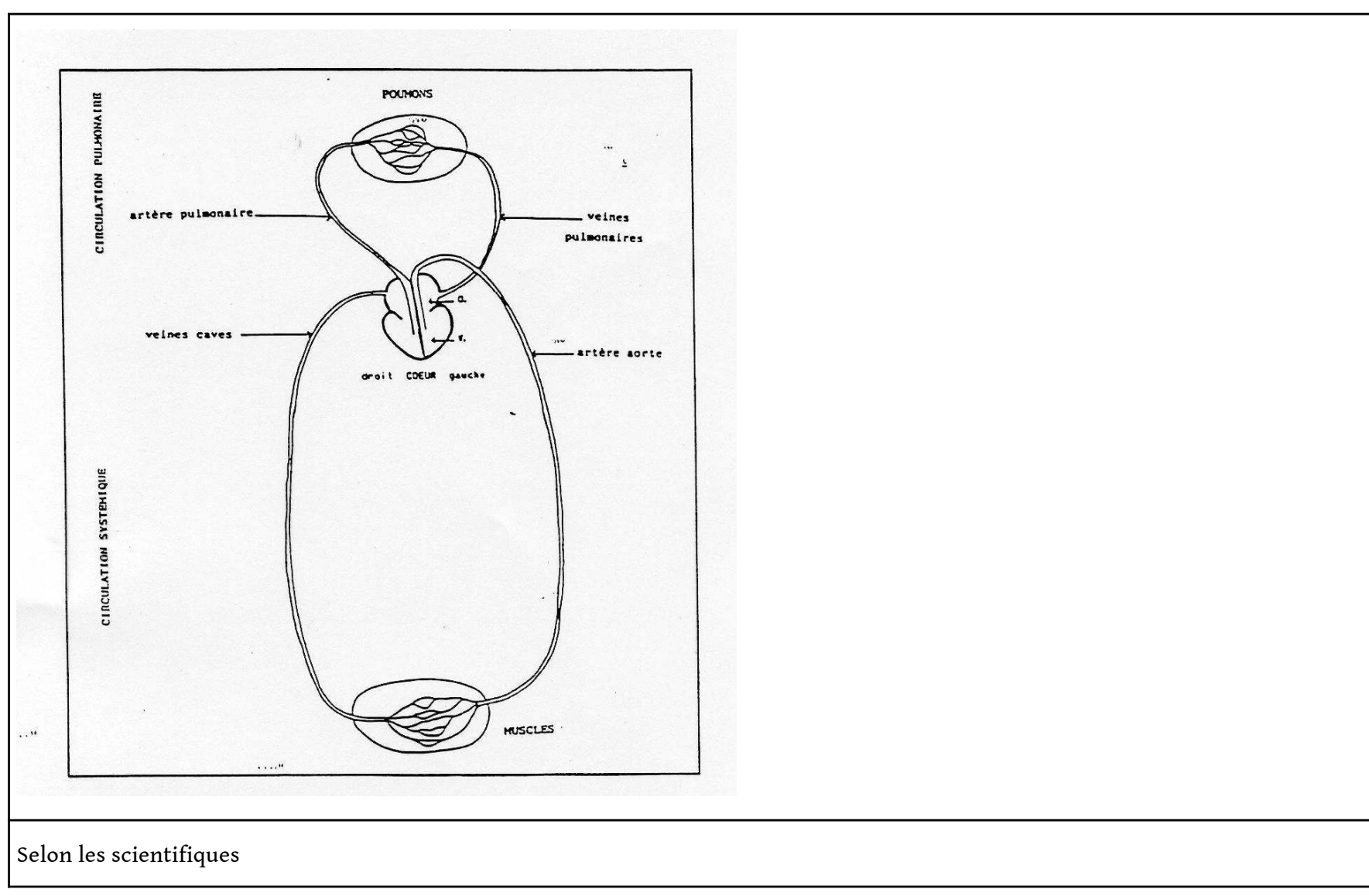




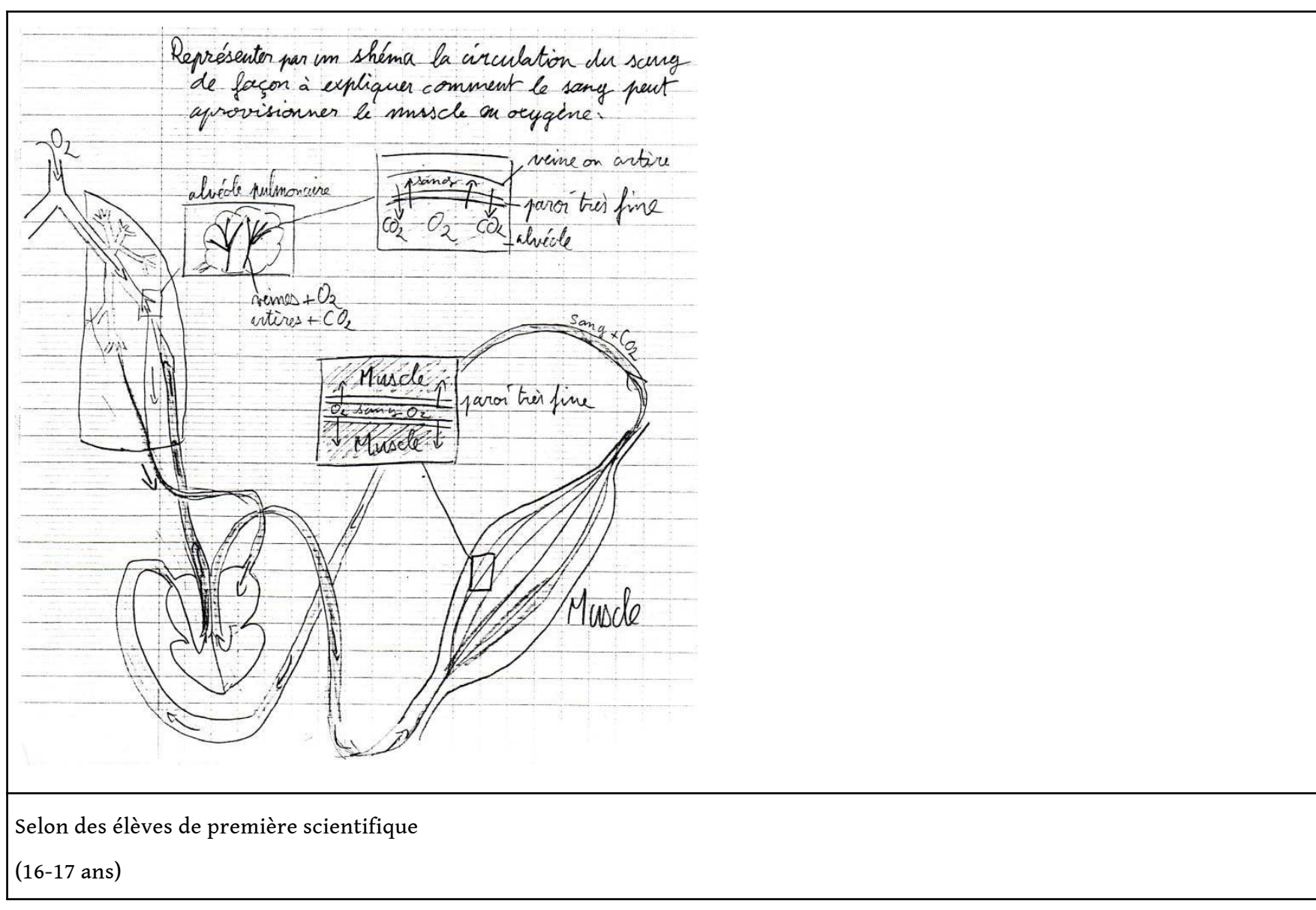

FiguRE 1. LA DOUBLE CIRCULATION SANgUINE double circuit, de façon à satisfaire les autres conditions de la nutrition des organes et de leurs cellules (approvisionnement en petites molécules organiques, élimination des déchets azotés), des divergences se font jour entre les modèles explicatifs qu'ils élaborent et ce que font les scientifiques. Classiquement, leurs productions écrites peuvent être rangées en deux grandes catégories.

Catégorie a. L'approvisionnement du muscle en nutriments et l'évacuation de ses déchets se fait par le placement du tube digestif (ou plus précisément de l'intestin) et des reins de telle sorte que le muscle reçoive au plus vite les nutriments dont il a besoin et élimine très rapidement les déchets qu'il produit (figure 2a). Tous les processus physiologiques (absorption intestinale, assimilation, excrétion) s'enchaînent dans des espaces différents. C'est une organisation en série des organes qui permet à ceux-ci de se nourrir efficacement et au sang de ne pas se salir.

Catégorie b. Le tube digestif (ou l'intestin) et les reins sont intégrés en parallèle dans le système circulatoire. Ils sont placés sur une dérivation vasculaire réunissant l'artère aorte aux veines caves (figure 2b). Si nous faisons fonctionner cette organisation, nous remarquons que les nutriments et les déchets produits par les muscles empruntent des trajets beaucoup plus longs que dans une disposition en série. De plus, l'atteinte des organes cibles peut résulter d'une variété de trajets. Prenons l'exemple des déchets: avant d'être évacués par les reins, ceux-ci passent nécessairement par le cœur, les 
poumons, et à nouveau le cœur. Ensuite, plusieurs directions s'offrent à eux : l'emprunt d'une dérivation de l'aorte (pas forcément celle des reins) ou à nouveau un passage par le muscle.

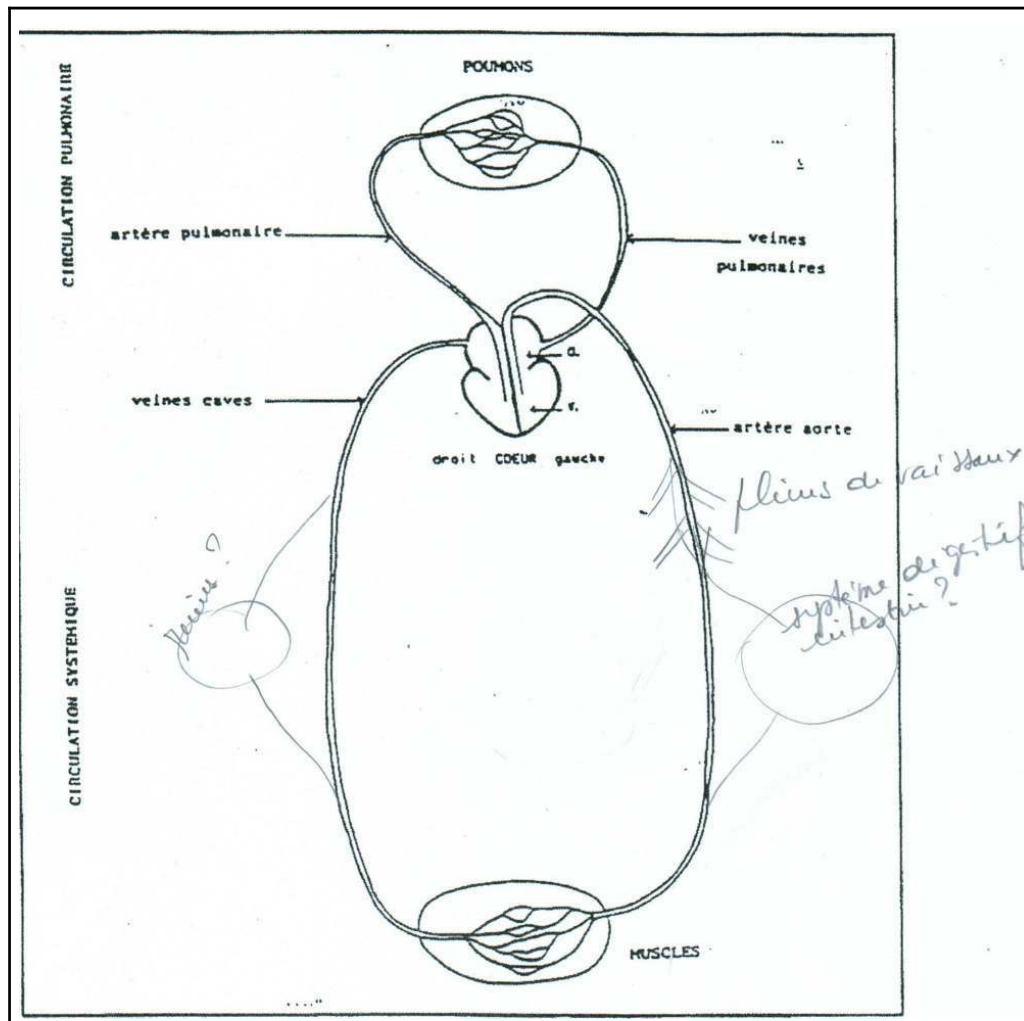

2a. Modèle en série 


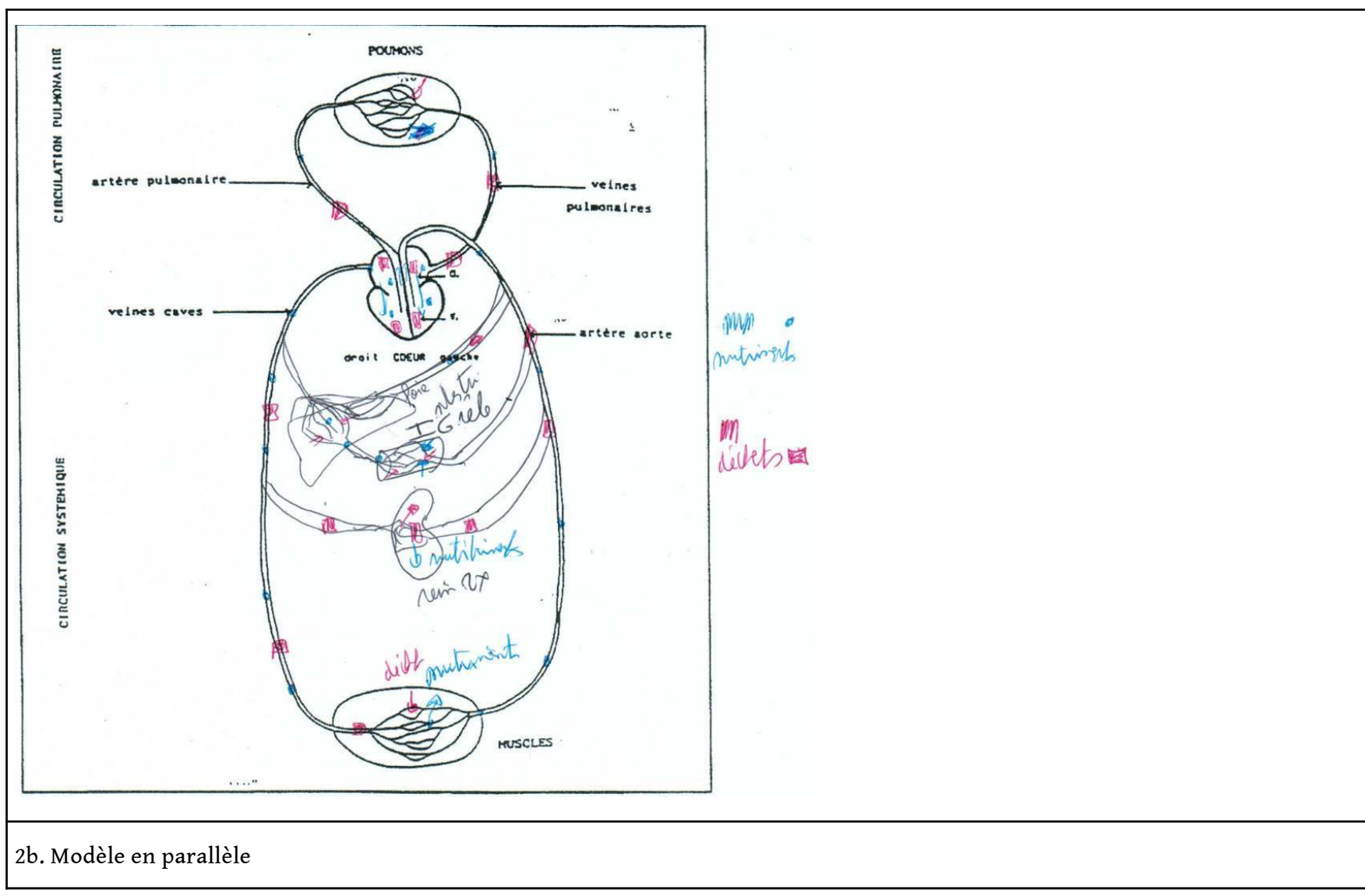

FIgURE 2. LES DEUX gRANDS TYPES DE MODÈLES D'APPROVISIONNEMENT DES MUSCLES EN NUTRIMENTS ET D'ÉVACUATION DE LEURS DÉCHETS

19 La catégorie b correspond à l'approche qu'ont les scientifiques de ce problème. Il s'agit d'une approche systémique, basée sur la prise en compte simultanée des processus en jeu, à savoir les entrées (nutriments) et les sorties (déchets) du système cardiovasculaires. Elle conduit à imaginer que le sang contient à la fois des nutriments et des déchets : il est donc en permanence et partout un peu «nutritif» et un peu "sale». L'important est que les taux de ces constituants s'inscrivent dans des fourchettes de valeurs ne compromettant pas la vie de l'organisme (les analyses de sang donnent à voir ces fourchettes de valeurs et ces seuils).

Cette manière d'expliquer (cette modélisation en « parallèle »), très rare chez le élèves, va à l'encontre de l'approche linéaire exprimée dans la catégorie a. Cette dernière rend compte de la préoccupation d'approvisionner les muscles en substances nutritives alimentaires et d'avoir du sang exempt de déchets. Elle confère aux nutriments et aux déchets le rôle de personnages en charge pour les premiers de satisfaire promptement les besoins des muscles et pour les seconds de disparaître au plus vite de façon à ce que le sang soit nettoyé. Cette modélisation relève d'une mise en «petite histoire ». Elle facilite la compréhension du fonctionnement de ce système complexe où plusieurs processus sont en jeu, évitant ainsi la difficulté de penser leur fonctionnement synchrone. Mais elle représente un véritable obstacle à la compréhension de sa stabilité structurelle (c'est-àdire que malgré un renouvellement permanent de matière, son organisation reste identique). 


\section{Evolution de la zone d'une dorsale océanique}

21 Cette situation de classe vient après que des activités ont permis à des lycéens d'une classe de première scientifique (16-17 ans) de se replacer dans le cadre de la théorie de la tectonique des plaques, d'établir les grands traits de l'histoire d'un fond océanique, et de réfléchir sur l'origine et la formation du magma qui alimente une dorsale. Pour les scientifiques, la zone dorsalienne représente en effet la limite entre deux plaques tectoniques divergentes. A son niveau, l'espace libéré par la dérive de chacune d'elles est aussitôt colmaté par la formation de nouveaux pans de plaques (on parle d'accrétion) par solidification du magma qui y parvient. C'est un autre exemple de système à stabilité structurelle dynamique.

Après qu'il est été présenté à des lycéens la coupe d'une dorsale océanique (une représentation déjà travaillée avec eux), il leur est demandé de prévoir comment sera cette zone dans quelques millions d'années. Pour répondre à la question posée, ils disposent d'une coupe de dorsale surnuméraire, qu'ils doivent aménager et compléter d'un petit texte explicatif.

L'enjeu est de voir comment ces élèves intègrent la tectonique des plaques et si, d'emblée, ils mobilisent ou non l'idée de stabilité structurelle de la zone axiale de la dorsale, c'est-àdire s'ils envisagent simultanément la dérive des plaques et la mise en place de nouveaux morceaux de lithosphère dans l'espace qu'elles libèrent.

Les réponses des élèves (coupe aménagée et texte accompagnateur produits avant apprentissage) se répartissent en deux grandes catégories, selon qu'elles prennent ou non en compte la stabilité structurelle de la zone de la dorsale.

\section{Catégorie 1. Une évolution sans stabilité structurelle du fait d'ajouts partiels}

La figure 3 illustre le type de réponse que proposent la plupart des élèves de première scientifique (16-17 ans). 


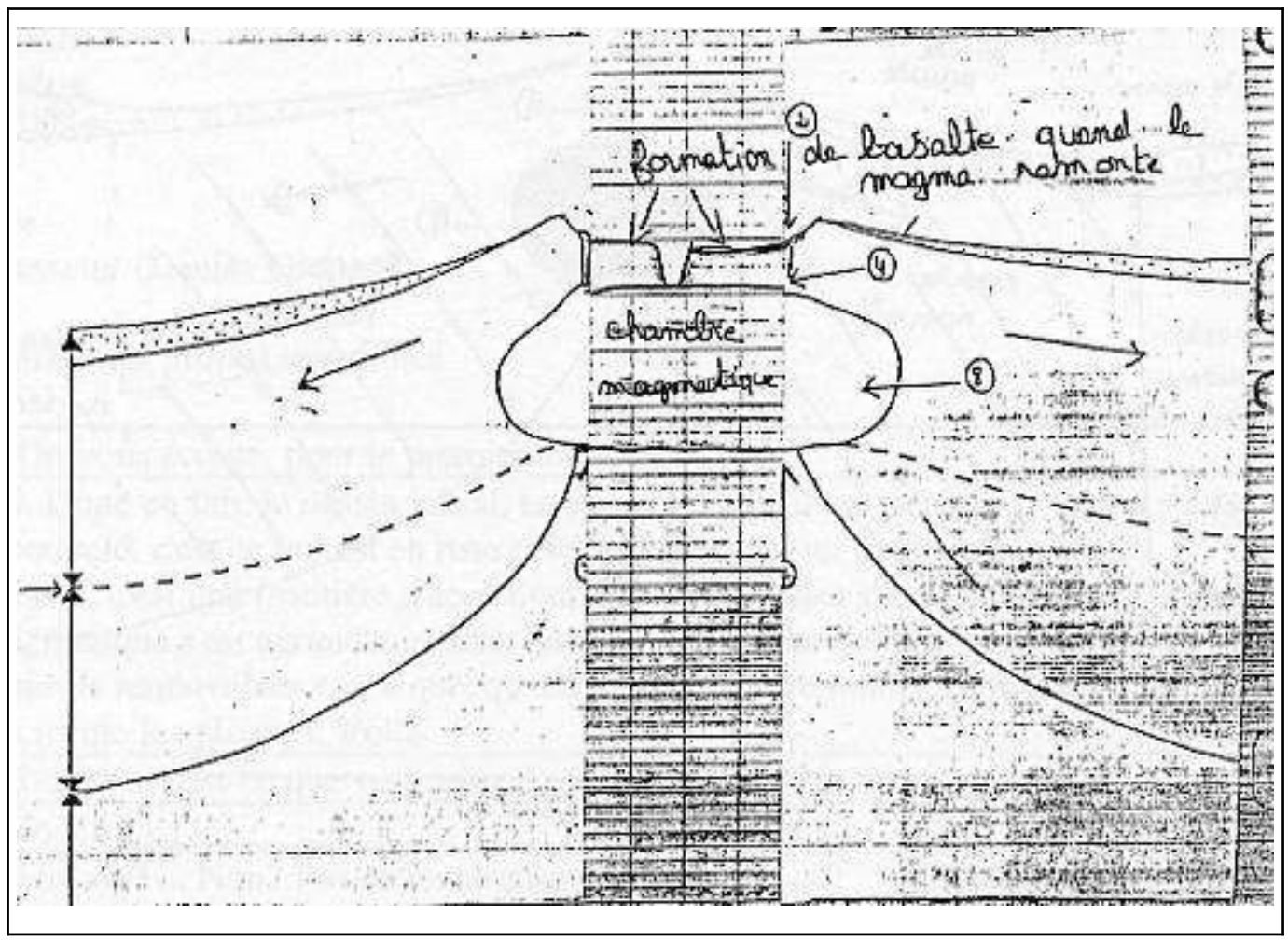

Figure 3. l'évolution sans stabilité structurelle de la zone d'une dorsale océanique (élève de première scientifique, 16-17 ans)

"La dorsale est une frontière de divergence donc les plaques s'écartent. Par conséquent la chambre magmatique s'agrandit. Et les plaques au niveau de la dorsale grâce à la formation de basalte lors de la remontée du magma. »

Dans une telle réponse, les élèves font intervenir deux éléments principaux (deux plaques) qui ont la propriété de s'écarter. L'évolution des plaques (1) ("les plaques s'écartent ») conduit à l'agrandissement de la chambre magmatique (2) («Par conséquent la chambre magmatique s'agrandit ») et à la mise en jeu d'un magma ascendant qui construit du plancher océanique (3) ("Et les plaques au niveau de la dorsale (s'agrandissent) grâce à la formation de basalte lors de la remonté du magma »).

Ce type de réponse d'élèves correspond à une petite histoire : il y a un commencement, une succession d'épisodes et une fin (provisoire puisque les plaques risquent de s'écarter à nouveau) à cette histoire ; la béance provoquée près de la surface par le déplacement des plaques crée un déséquilibre qui remet en cause la permanence des plaques dans cette zone. Cet "accident» est implicite dans le discours des élèves. L'intervention d'un nouveau personnage, le magma, permet de l'inférer. Celui-ci est mobile verticalement et «à disposition" dans la chambre magmatique qui permet un rétablissement de la situation par colmatage du trou près de la surface (vs une accrétion sur toute l'épaisseur des plaques lithosphériques).

Ainsi, alors qu'il faudrait prendre en compte simultanément deux processus (la dérive et l'accrétion des plaques), ces élèves les font intervenir de manière successive, en restreignant l'accrétion à la zone proche de la surface. Et l'enchaînement des épisodes fonctionne par un syncrétisme de temps et de causalité. Au "en même temps " se substitue un « et » ou « et donc » (une chronologie associée à un déplacement et/ou une causalité) : les plaques s'écartent « et/donc » du magma remonte « et/donc » des plaques 
s'agrandissent «et/donc» les plaques s'écartent, etc. Cela donne un fonctionnement saccadé de la zone de la dorsale et conduit à une chambre magmatique démesurée.

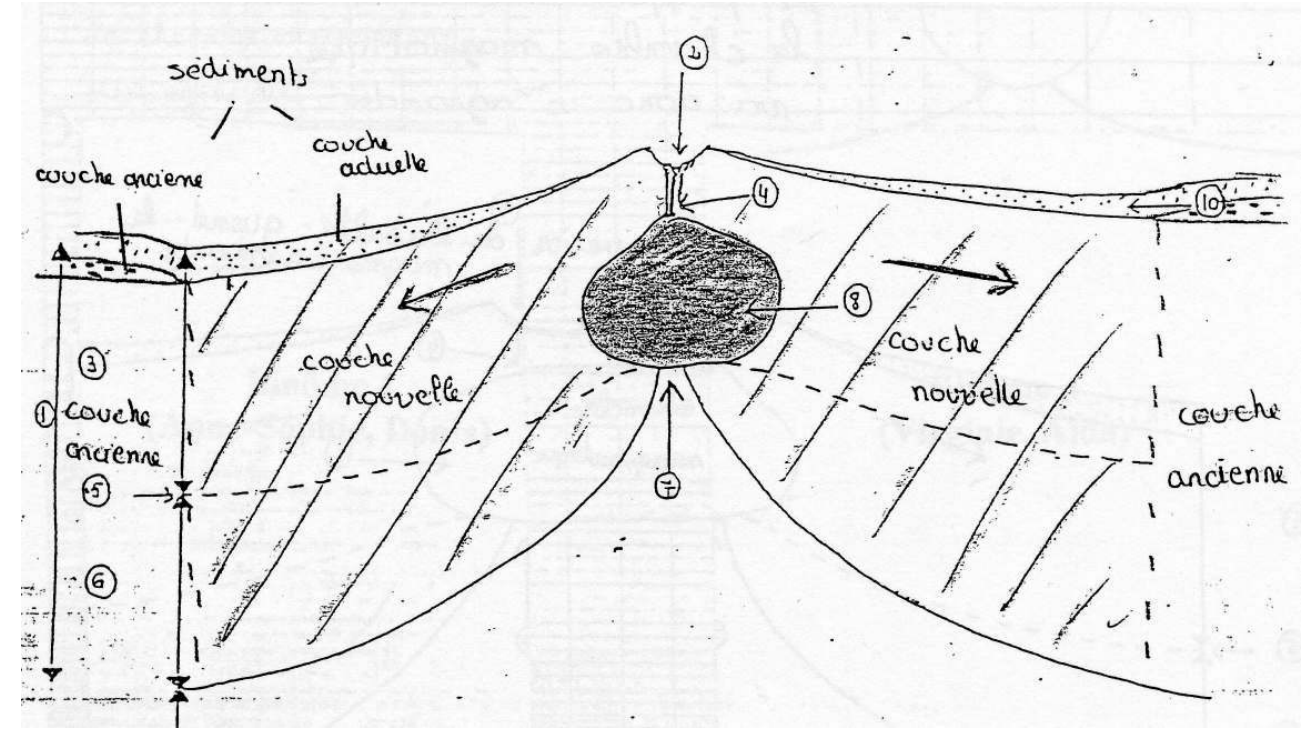

Figure 4. L'évolution avec stabilité structurelle de la zone d'une dorsale océanique (élève de première scientifique, 16-17 ans)

" Dans quelques millions d'années, l'océan se sera agrandi puisque les plaques s'écartent et il y aura une ou plusieurs autres couches de sédiments par-dessus ceux qui s'y trouvent actuellement. Le rift lui ne va pas s'agrandir puisqu'en s'écartant le magma sort et se refroidit en formant une croûte, de plus si le rift s'agrandissait aujourd'hui, le rift serait géant puisqu'au départ là où il y a certains océans avant il n'y en avait pas. La chambre magmatique sera tel quel »

Comme dans la réponse précédente (figure 3), il est possible de définir des personnages principaux : «les plaques s'écartent »). De la même manière, les élèves ne nous disent rien d'un quelconque espace libéré par le mouvement divergent des plaques. Mais ils rendent compte explicitement des contraintes pesant sur l'histoire: "Le rift lui ne va pas s'agrandir»; "la chambre magmatique sera telle quel». Contraintes qu'ils respectent en mettant en jeu la simultanéité de deux processus qui se compensent : «puisqu'en s'écartant (il s'agit du rift, partie supérieure des plaques) le magma sort et se refroidit en formant une croûte ». La béance n'a pas lieu d'être. Ce groupe d'élèves construit la stabilité structurelle en montrant, par une expérience de pensée, qu'il a une conscience aiguë de certaines impossibilités. Il les exprime et il les fixe, dans une articulation du passé et de l'avenir: "si le rift s'agrandissait aujourd'hui, le rift serait géant puisqu'au départ là où il y a certains océans avant il n'y en avait pas ".

L'histoire produite par ces élèves est beaucoup plus complexe que la précédente. Au lieu d'enchaîner des processus, ils les mettent à l'œuvre simultanément. Ce faisant, ils " cassent » la petite histoire au profit d'une "non histoire », puisqu'en définitive cette zone conserve les mêmes caractéristiques au cours du temps. De plus ces élèves étayent leur explication par des contraintes fortes (exemple : le rift a toujours la même taille). Enfin, ils déploient des expériences de pensée sur des possibles et des impossibles explicatifs qu'ils articulent à la construction d'un registre empirique plus fourni. 


\section{Conclusion}

32 Pour des problèmes biologiques et géologiques fonctionnalistes, l'accès à des savoirs scientifiques apodictiques se heurte à l'inclination des élèves à produire des «petites histoires » explicatives. Le passage à une pensée scientifique exige qu'ils se défassent d'un cantonnement sur les propriétés des objets constitutifs des systèmes étudiés et sur des raisonnements linéaires causaux simples, et qu'ils s'approprient des modes de pensée nouveaux, au premier rang desquels nous plaçons les raisonnements systémiques et des formes explicatives du temps plus élaborées que la chronologie.

\section{Deuxième sorte de dépassement de récits ordinaires en sciences : la construction du temps long producteur de phénomènes et de nécessité événementielle}

\section{L'histoire des systèmes biologiques et géologiques}

A la préoccupation des scientifiques d'étudier le fonctionnement des systèmes biologiques et géologiques, s'ajoute celle d'établir leur histoire (histoire évolutive des vivants, histoire de la Terre). Ces problèmes historiques participent également de la formation des élèves. Or ces reconstitutions historiques propulsent dans des temporalités dépassant largement la présence humaine sur la planète, ce qui impose des contrôles théoriques et méthodologiques forts pour garantir la scientificité des récits produits. Le raisonnement fondé sur le principe de l'actualisme y contribue. Il repose sur le transfert de causes actuelles (volcanisme, érosion, sédimentation, métamorphisme, phénomènes compressifs et distensifs, etc.; Gohau, 1997, p. 140) aux événements passés. Etant «le pont qui permet à notre imagination de se transporter du présent jusqu'au passé et d'évoquer, avec une certaine confiance dans son exactitude, la vision de faits qu'aucun œil humain n'a contemplés ». (Hooykaas, 1970, p.11), il contribue à la construction raisonnée de phénomènes historiques (Orange-Ravachol, 2003 ; Orange-Ravachol \& Beorchia, 2011). Si l'actualisme a un rôle majeur dans l'activité scientifique, il semble que les élèves n'en usent pas spontanément, ou y recourent dans sa version peu élaborée (actualisme d'analogie simple; Orange Ravachol, 2003) et que, sans une telle vigilance, leurs propositions versent dans de " petites histoires ». C'est ce que nous allons voir dans le cadre de deux problèmes géologiques.

\section{La formation d'une chaîne intracontinentale de montagnes}

Le problème de la formation des chaînes de montagnes (Alpes, Himalaya) est difficile, y compris pour les géologues. Son travail exige qu'ils établissent des relations complexes entre le passé et le présent, via le principe méthodologique de l'actualisme : ils identifient des phénomènes actuels pouvant participer de l'explication (des compressions océaniques par exemple) qu'ils exportent dans des temps anciens (actualisme de premier niveau ou actualisme d'analogie). Mais cela ne suffit pas, car la nature actuelle ne donne pas à voir l'intégralité d'une surrection de reliefs (orogenèse) et la mise en place des structures tectoniques associées (plis, failles). Elle n'en fournit que des bribes. C'est par 
l'intégration de ces bribes sur un temps long (actualisme à temps long constructeur de phénomènes, figure 5) que les géologues produisent un phénomène géologique nouveau. Ce faisant, ils ne cèdent pas à un catastrophisme naïf, c'est-à-dire à des causes conduisant à des transformations faciles. Ils ne limitent pas non plus les fonctions du temps à de la chronologie et de la durée "magique ", capable de tout permettre, ils construisent la nécessité d'un temps long producteur de nouveaux phénomènes (orogenèse et tectogenèse).

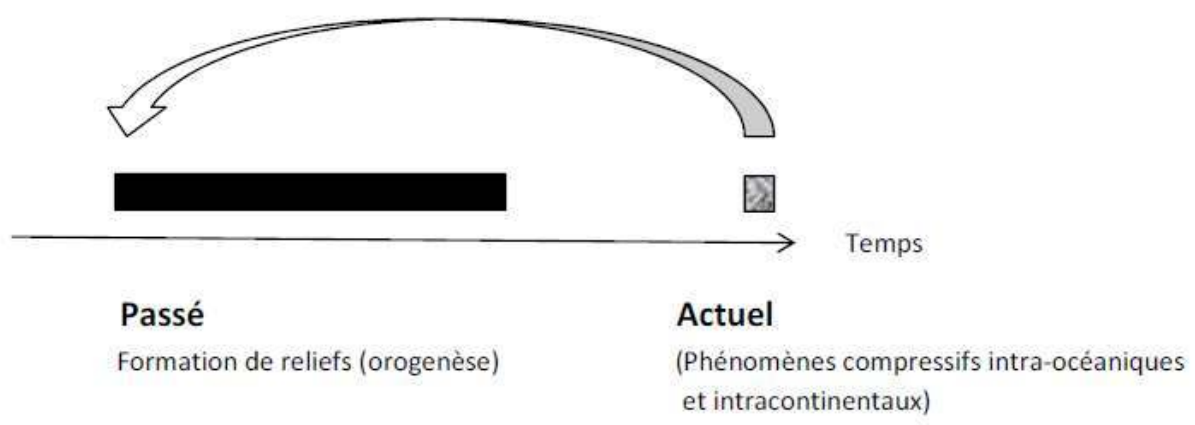

Nécessité d'un temps long constructeur de phénomènes

Figure 5. Actualisme à temps long et formation d'une chaîne de montagnes (orogenèse), (Orange Ravachol, 2003, 2012)

Fort de ces éclairages épistémologiques, venons-en à ce que font les élèves. Le cas que nous étudions concerne des collégiens de quatrième (13-14 ans). Après qu'un rapide rappel des notions acquises sur la tectonique des plaques a été fait (le renouvellement des plaques lithosphériques au niveau des dorsales et leur disparition dans les zones de subduction), le professeur présente la chaîne de montagnes himalayenne (photos satellite), la situe sur un planisphère et pose à la classe le problème de sa formation. La consigne donnée aux élèves est la suivante: «Expliquez comment s'est formée une chaîne de montagnes telle que l'Himalaya. Vous répondrez par un texte et des schémas mettant en jeu la lithosphère ». Ceux-ci s'en emparent, d'abord individuellement, puis en groupes que l'enseignant a voulu homogènes du point de vue des explications. La figure 6 illustre le type de réponse que produisent les groupes. 


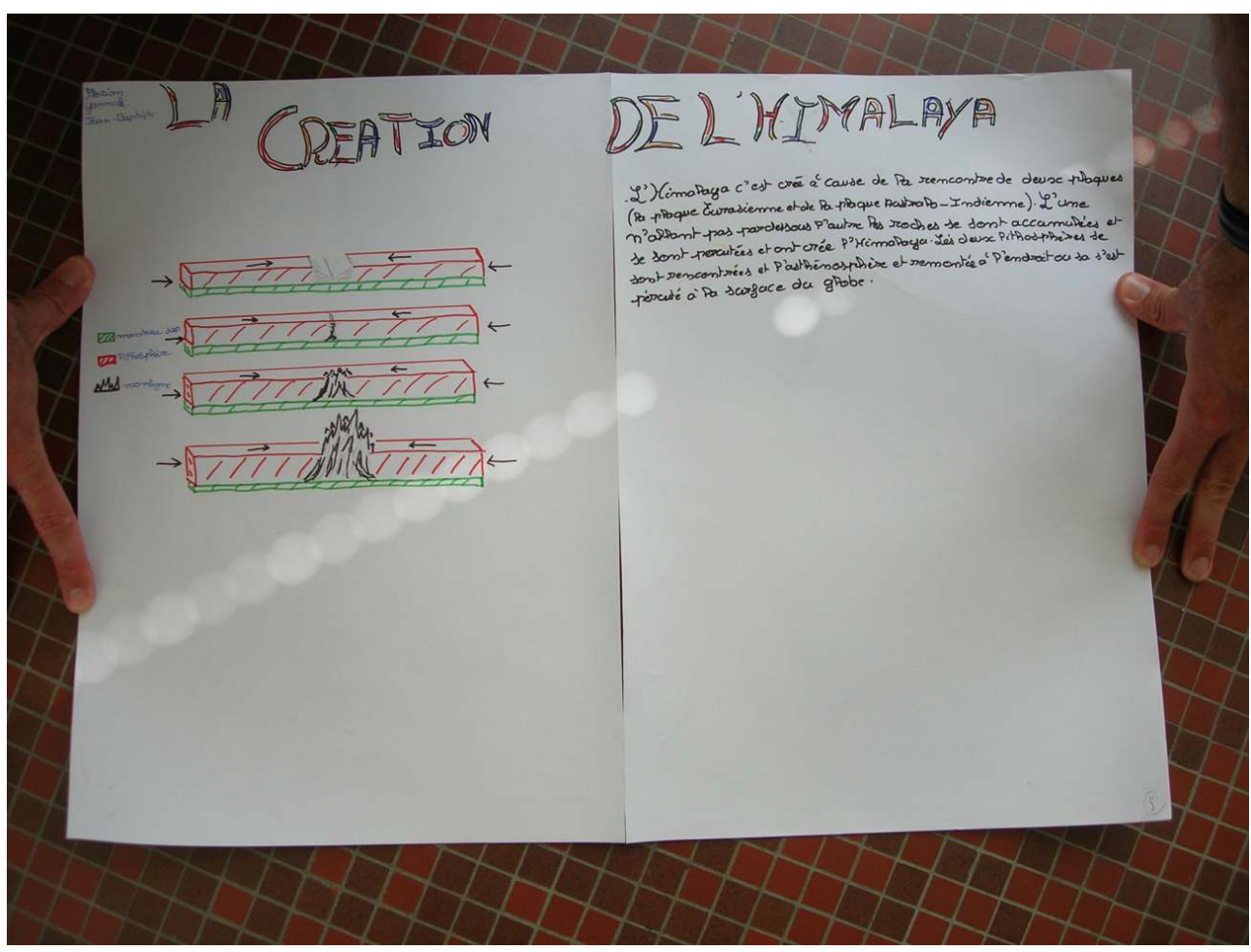

Figure 6. La formation de l'Himalaya (élèves de 13-14 ans)

"L'Himalaya s'est créée à cause de la rencontre de deux plaques, la plaque eurasienne et la plaque australoindienne. L'une n'allant pas par dessous l'autre les roches se sont accumulées et se sont percutées et ont créé l'Himalaya. Les deux lithosphères se sont rencontrées et l'asthénosphère est remontée à l'endroit où ça s'est percuté à la surface du globe. »

Tous les élèves font intervenir des plaques dont les déplacements aboutissent à une collision provoquant une surrection montagneuse. La figure 6 en donne un exemple : des plaques initialement éloignées, donc séparées par une sorte de grand trou, se rapprochent et se cognent. L'édifice montagneux résulte d'un pincement avec remontée de l'asthénosphère, le matériau sous-jacent, mais ce peut être aussi d'une courbure vers le haut des plaques, ou bien encore le passage d'une des deux plaques sur l'autre, ce qui provoque un bombement en surface. Ce faisant, 1) les élèves conservent l'intégrité des plaques (comme des personnages immuables d'une histoire) ;2) ils attribuent aux plaques la mobilité d'« autos-tamponneuses » et les font jouer dans un cadre spatio-temporel peu contraignant. Ce sont de petites histoires inventées (storytelling) de plaques : elles usent d'un raisonnement séquentiel: un élément du système prend valeur de personnage principal dont on suit les aventures; elles exacerbent les propriétés de mobilité et de rigidité des plaques; elles fonctionnent sans apparent contrôle actualiste et empirique. Ainsi les élèves restent cantonnés dans la pensée commune et traitent donc la question comme un problème de fonctionnement dans un temps et avec des objets ordinaires.

Ces petites histoires ne correspondent pas aux explications scientifiques actuelles. Les collégiens raisonnent plus dans un cadre de dérive des plaques que de tectonique des plaques : ils considèrent les plaques comme des objets séparés qui se cognent quand les géologues les décrivent comme occupant toute la surface terrestre, donc en permanence jointives ; ils s'en tiennent à expliquer la formation du relief (sans réelle tectogenèse) par des mouvements plaquistes contingents (ils pourraient être autres) et non assujettis à des contraintes spatiales et temporelles exigeantes; ils recourent ainsi à un catastrophisme naïf. S'il y a bien référence à l'actuel (existence de plaques par exemple), il ne s'agit que 
d'un actualisme de premier niveau, banal, qui ne fait pas intervenir un temps long créateur de phénomènes.

\section{La disparition de la plupart des dinosaures}

Lorsque les géologues expliquent la formation d'une chaîne de montagnes (orogenèse et tectogenèse), ils en font un phénomène et non un événement, en se situant dans le cadre d'un actualisme de temps long. Ce faisant, à condition d'être contrôlé par le principe de l'actualisme, le travail du problème historique (la problématisation historique) peut se ramener au travail d'un problème de fonctionnement (une problématisation fonctionnaliste) mettant en jeu un temps long. Cependant, pour d'autres questions de géologie ou de biologie historique, il leur devient quand même nécessaire d'introduire des événements. Ainsi, par exemple, pour rendre compte d'extinctions de masse comme celle de la fin du Crétacé où la plupart des Dinosaures disparaissent (ou pour penser l'origine de la vie terrestre) : il n'existe pas d'équivalents actuels connus.

La nécessité de traiter cette extinction comme un événement singulier est renforcée si, dans le cadre évolutionniste, elle est pensée comme une condition de possibilité du développement des Mammifères donc des Hominidés. Dans ce cadre, l'événement « disparition », s'il est compris comme une condition de possibilité du développement de la lignée mammalienne, est rendu nécessaire du fait même que l'Homme existe, c'est-àdire dans une logique de remontée de l'histoire révolue (figure 7). La problématisation d'un tel événement procède donc d'un double jeu: d'une part, en suivant le cours de l'histoire, d'une exploration et d'une articulation des possibles (que pourrait-on avoir d'autre ?) et d'autre part d'une propagation à rebrousse-temps des nécessités à partir d'un état postérieur. La contingence associée à l'événement tient donc au fait que d'autres possibles auraient pu exister ou peuvent exister dans une expérience de pensée qui consiste à ré-enrouler le temps et à le dérouler à nouveau (Gould, 1991). Cette nécessité événementielle intervient donc dans la problématisation historique pour autant que l'événement est tenu comme une condition de possibilité d'un autre événement, postérieur, dont la contingence mérite d'être questionnée. 


\section{Remontée de l'histoire}

\section{Descente de l'histoire}

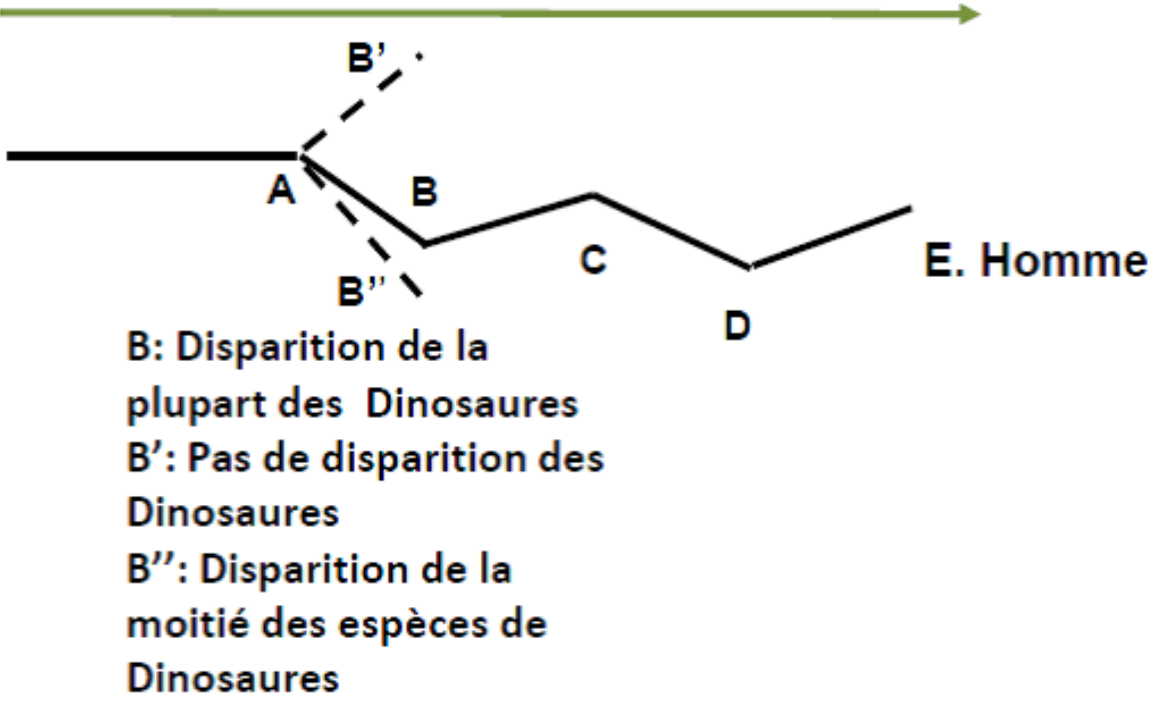

Figure 7. Problématisation historique, contingence et nécessité événementielle (Orange Ravachol, 2012)

Lorsque nous mettons des élèves en situation de reconstituer l'histoire des vivants (l'origine de la vie sur la Terre, les crises biologiques), nombreux sont ceux qui se placent dans un cadre évolutionniste transformiste, où des êtres microscopiques simples (bactéries, etc.) se transforment au cours du temps en êtres vivants plus gros et plus complexes. Les métamorphoses des êtres vivants règnent en maitre : ce sont elles qui introduisent de la complexité et qui négligent le temps. La figure 8 fournit l'exemple de réponse d'un élève de terminale scientifique (17-18 ans). C'est une petite histoire où, en l'absence de règles, sinon l'existence d'une échelle des êtres en toile de fond, quasiment tous les changements et toutes les transgressions sont possibles: «La mythologie de la métamorphose (...) sert de toile de fond à une mythologie évolutionniste qui fonctionne toujours-à nouveau comme obstacle à l'assimilation des conceptions darwiniennes » (Rumelhard, 1995, p. 336).

41 Pour ce problème historique, les élèves mettent en jeu des « objets » (ici les êtres vivants) à valeur de personnages soumis aux aléas de l'existence et dont des propriétés particulières permettent leur adaptation par transformation. Au contraire des chercheurs qui, dans une logique rétrospective, font de l'origine de la vie un événement nécessaire, les élèves raisonnent en descendant l'histoire de la vie, en privilégiant des changements de formes qu'ils ne questionnent pas. Si quelques-uns procèdent en remontant l'histoire de la vie, ils le font en s'appuyant sur leur expérience d'Homme (reproduction sexuée mise en jeu à l'infini) qui confine rapidement à l'absurde (ils ne trouvent pas d'origine). 


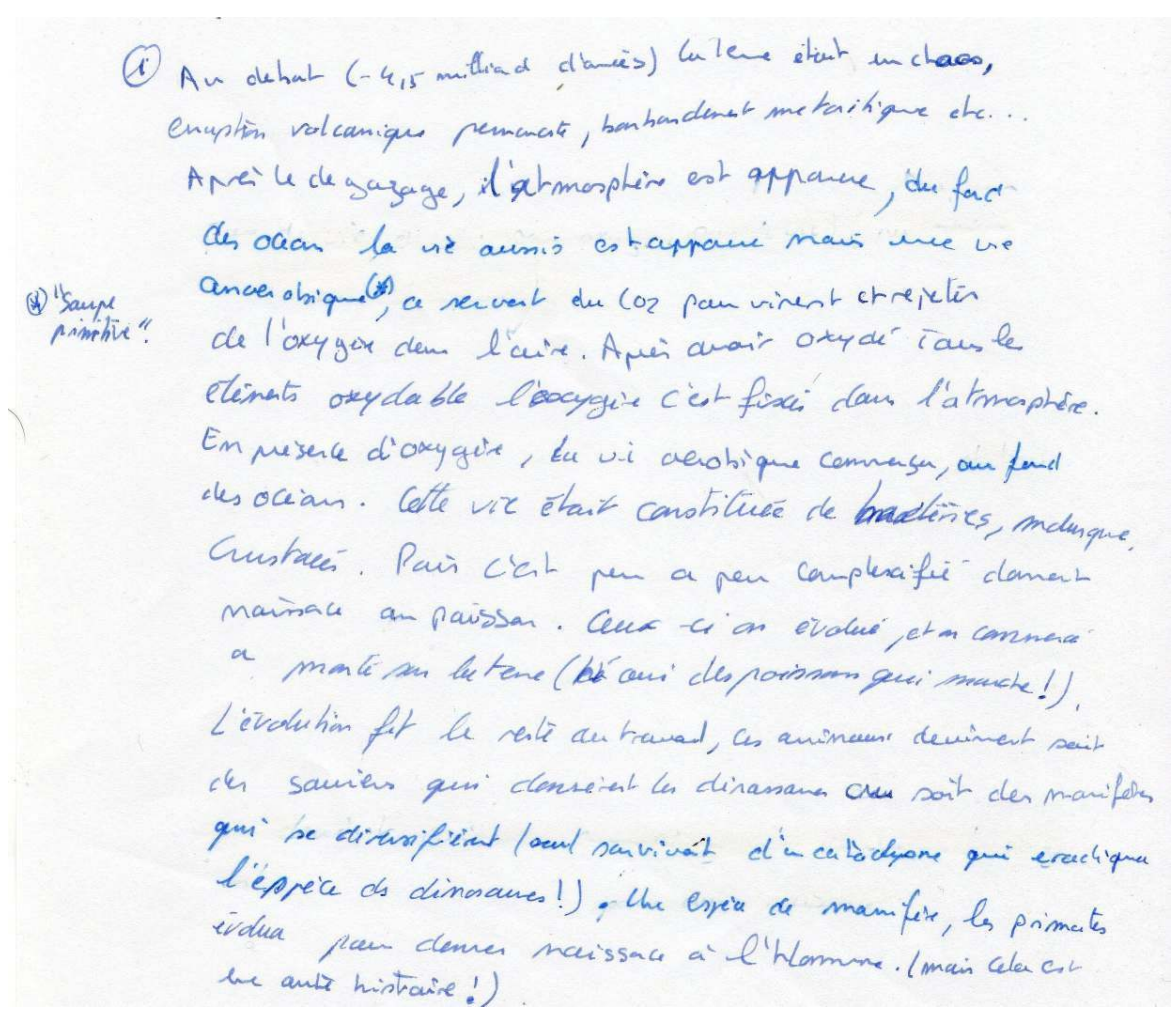

Figure 8. L'origine et l'évolution de la vie sur la Terre (élève de terminale scientifique, 17-18 ans) "Au début (-4,5 milliard d'années), la Terre était un chaos, éruptions volcaniques permanentes,

bombardements météoritiques etc. Après le dégazage, l'atmosphère est apparue, du fait des océans la vie aussi est apparue, mais une vie anaérobique $(*)$, se servant du CO2 pour vire et rejeter de l'oxygène. Après avoir oxydé tous les éléments oxydables, l'oxygène s'est fixé dans l'atmosphère. En présence d'oxygène, la vie aérobique commença, au fond des océans. Cette vie était constituée de bactéries, mollusques, crustacés. Puis s'est peu à peu complexifiée donnant naissance au poisson. Ceux-ci ont évolué et ont commencé à monter sur la Terre (hé oui des poissons qui marchent!). L'évolution fit le reste du travail, ces animaux devinrent soit des sauriens qui donnèrent les dinosaures ou soit des mammifères qui se diversifièrent (seuls survivants d'un cataclysme qui éradiqua l'espèce des dinosaures !). Une espèce de mammifère, les primates évolua pour donner naissance à l'Homme. (mais cela est une autre histoire!)»

* «soupe primitive»

\section{Conclusion}

La biologie et la géologie " parlent » aux élèves mais ceci est à double tranchant. Si cela leur permet de s'engager dans des propositions explicatives, cela cultive aussi leur habitude intellectuelle de mise en histoire. Car spontanément les élèves font du storytelling et produisent des récits ordinaires: mise en jeu d'objets dont on suit les aventures contingentes (elles pourraient être autres); enchaînement d'épisodes où temps et causalité jouent de manière syncrétique; introduction d'événements ad hoc. C'est une façon simple, souple et confortable de régler les questions fonctionnalistes et historiques sans entrer dans la problématique phénomène/événement et dans la complexité des formes explicatives du temps, dont les instructions officielles et les manuels ne sont d'ailleurs pas avares. Mais les élèves ne sont pas dupes, car ils se disent aussi démunis et en mal de se représenter ce qui est possible. Aussi faut-il voir les mises en histoire qu'ils réalisent comme du bricolage (Levi Strauss, 1962) auquel ils ont recours, plus par nécessité de produire une réponse que par adhésion à ce qu'ils proposent (Orange Ravachol, 2012, p. 95). Où l'on retrouve l'intelligence du sens commun et de ses récits qui construisent de la cohérence en assemblant du disparate... Où l'on comprend la nécessité 
de doter les élèves de ressources théoriques et méthodologiques pour s'en dégager. La prise d'importance des "Éducations à ", qui anthropise fortement les problèmes scientifiques étudiés à l'Ecole, ne fait qu'accentuer cette nécessité.

\section{BIBLIOGRAPHY}

Adam, J.-M. (1984). Le récit. Paris : PUF, collection Que-sais-je?

Astolfi,J.-P. (2008). La saveur des savoirs, Disciplines et plaisirs d'apprendre. Paris : ESF Editeur.

Bachelard, G. (1971). Epistémologie, Textes choisis par Dominique Lecourt. Paris : PUF.

Bachelard, G. (1938). La formation de l'esprit scientifique. Paris : Vrin.

Bruner, J., (2002). Pourquoi nous racontons-nous des histoires ?. Paris : Retz

Bruner J. (1997). ... Car la culture donne forme à l'esprit. Paris : Eshel (1991).

Bruner, J. (1996). L'éducation, entrée dans la culture. Paris : Retz.

Fabre, M. \& Orange, C. (1997). Construction des problèmes et franchissements d'obstacles. ASTER, 24, 37-57. (disponible sur : http://documents.irevues.inist.fr/handle/2042/8550, consulté le 15 septembre 2017)

Gould, S. J. (1991). La vie est belle, Les surprises de l'évolution. Paris : Seuil.

Gohau, G. (1997). Naissance de la méthode « actualiste » en géologie. In GOHAU G., dir., (1997). De la géologie à son histoire, CTHS, pp. 139-149.

Hooykaas, R. (1970). Continuité et discontinuité en géologie et biologie. Paris : Seuil.

Jacob, F. (1981). Le jeu des possibles. Paris: Librairie Fayard.

Latour B. \& Woolgar S. (1988). La vie de laboratoire. Paris : Éd. La Découverte.

Lévi-Strauss, C. (1962). La pensée sauvage. Paris : Plon.

Orange Ravachol, D. (2012). Didactique des SVT, Entre phénomènes et événements. Rennes : PUR, collection Païdeia.

Orange Ravachol, D. (2003). Utilisations du temps et explications en Sciences de la Terre par les élèves de lycée : étude dans quelques problèmes géologiques. Thèse de doctorat, Université de Nantes. (disponible sur : http://tel.archives-ouvertes.fr/, consulté le 15 septembre 2017)

Orange Ravachol, D. \& Beorchia, F. (2011). Principes structurants et construction de savoirs en sciences de la vie et de la Terre. Education et Didactique, vol.5, $\mathrm{n}^{\circ} 1, \mathrm{pp} .7-27$.(disponible sur : https://educationdidactique.revues.org/1016, consulté le 15 septembre 2017)

Piaget, J. \& Garcia, R. (1983). Psychogenèse et histoire des sciences. Paris : Flammarion.

Popper, K. (1991). La connaissance objective. Paris : Aubier (1972)

Popper, K. (1985). Conjectures et réfutations. Paris : Payot.

Rumelhard, G. (1995). Permanence, métamorphose, transformation. Biologie-Géologie, n², pp. 333-345. 


\section{ABSTRACTS}

Popper écrit que ce que nous appelons « science » se distingue des mythes qui l'ont précédée non parce qu'elle en est différente dans la forme (il s'agit bien toujours de raconter une histoire qui explique un phénomène) mais parce qu'il s'y ajoute un corrélat, la prise en compte critique de cette histoire par la discussion et l'empirie. Plutôt donc que d'opposer récit et explication, nous choisissons d'interroger, dans quelques problèmes des de la nature et en comparaison de ce que font les scientifiques, les "mises en histoire" des élèves et leur évolution dans un contexte favorisant le développement d'argumentations (débat scientifique ou entretien) : comment prennent-ils en charge les phénomènes, quelle utilisation font-ils des événements?

\section{INDEX}

Mots-clés: actualisme, biologie-géologie, élève, phénomène-événement, problématisation, récit

\section{AUTHOR}

\section{DENISE ORANGE RAVACHOL}

Professeur des Universités en sciences de l'éducation, (didactique des sciences et des « Educations à »), Université Lille 3, Theodile-CIREL, denise.orange@univ-lille3.fr 